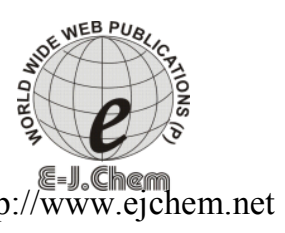

ISSN: 0973-4945; CODEN ECJHAO

E-Journal of Chemistry

2012, 9(2), 659-668

\title{
Synthesis of Silica Nanostructures and Optimization of their Size and Morphology by Use of Changing in Synthesis Conditions
}

\author{
A. ELSAGH \\ Faculty of Chemistry, Department of Chemistry \\ North Tehran Branch, Islamic Azad University, Iran \\ a_elsagh@iau-tnb.ac.ir \\ Received 10 October 2011; Accepted 31 December 2011
}

\begin{abstract}
The aim of the present research was optimization of practical conditions of the sol-gel synthesis. In so doing, silica particles were synthesized using sol-gel method and their size and morphology were investigated by use of SEM and TEM images. The effect of changing molar ratio of reactive including acids and silica pre-matter on the particles' morphology and size was studied. Also, acid type and silica pre-matter used in reaction was examined and the result product of the reaction were investigated in the presence of several acids and two types of silica pre-matter. The reaction time was studied as a very important factor in products' digestion phase which plays a significant role in determining particles' size and rate of cracking products. Finally, in optimized conditions $50-80 \mathrm{~nm}$ diameter nanostructures were synthesized. These products can be used as drug delivery systems.
\end{abstract}

Key words: nanostructures, sol-gel, morphology, electron microscope.

\section{Introduction}

During the past decade, many efforts have been done to develop new materials, especially nanostructure materials. It is proved that Nano-meter particles have some unique properties due to the small size and high specific surface area; similarly, mesoporous cases have many applications due to high internal surface area and small pore size. If nanometer particles (semiconductors or metals) enter into the cavities of porous materials, they will form a new material that has certainly some of the unique properties of Nano-particles and porous ${ }^{1}$. Porous structures have some optical, electrical and mechanical unique properties in nanometer dimensions, which can be applied in a wide range of applications such as photonics and electronics to the biological and medical sensors. Synthesis of porous particles with dimensions less than micrometer is very important to develop technologies ${ }^{2}$. Since the discovery of porous silica there is most focus to factor them due to their large 
surface area, uniform distribution of pore size and adjustable pore size between $1.5-30 \mathrm{~nm}^{3}$. Mesoporous silica has been attended as catalyst and adsorbent due to uniform structure of the cavity is capable to accept of relatively large molecules that Zeolite can include them. They also can be used to produce Nano-conductor and magnetic wires ${ }^{4}$. Sol-gel method today is known as a very suitable and commonly used method for preparing a variety of ceramic and non-ceramic materials. For the silica material, the most famous process includes hydrolysis of silicon dioxide and polymerization and getting hunk ${ }^{5-6}$. The obtained products features entirely depend on their synthesis conditions ${ }^{7}$. Many studies have been done on the relationship between preparation conditions and characteristics of obtained products $^{8-9}$. It is clear that the spatial structure of these materials made in successive steps ${ }^{10}$. The network includes pre-cursers or initial structures that in comparison with the final structure are much smaller than 100 nanometers with high difficult efficiency are ${ }^{11-12}$. But in this article a good method for the synthesis of mesoporous silica nanoparticles with high efficiency and controlled particle size between 50 to $80 \mathrm{~nm}$ has been reported that can be a good host for lanthanide complexes ${ }^{13-14}$.

\section{Experimental}

Lanthanum nitrate hexahydrate with P.a. purity degree was supplied from Sigma Aldrich Company. Tetraethylorthosilicate (TEOS) with $98 \%$ purity and Glacial acetic acid, were prepared from Merck Company Germany. Other chemicals used, with the purity of P.a. were prepared from Merck Company Germany. In this study non-ion water produced by Milli.RO.15 machine was used. Images of samples were prepared by the machine SEM model XL.30 made by Philips Co, Japan and TEM, model FEG.CM.20, Philips, made in Japan to identify the Nano-materials. The centrifuge model Rotofix.32 Hitachi Company was also used to isolate the reaction product synthesized from the environment in the Atomic Energy Organization of Iran.

\section{Synthesis of prototypes according sol-gel method}

Prototypes were obtained by mixing acetic acid, water and (TEOS) with 9, 1.6, and $4.5 \mathrm{ml}$ values respectively (Table 1). Mixture was stirred for 30 seconds. Then it took $6 \mathrm{~h}$ to complete the reaction, and the gel formed at the beginning of the reaction, is complete. Then, the product formed by centrifugation with $6000 \mathrm{rpm}$ went away from a reactive environment. In order to remove initial material with no reaction from the surface of the formed particles, the product was washed four times with $20 \mathrm{ml}$ ethanol and went away by the centrifuges ${ }^{13}$. Then the samples were examined by SEM (Figure 1).

Table 1. Synthesis of samples according to sol-gel general method.

Deionized water, $\mathrm{mL}$

1.6
TEOS, $\mathrm{mL}$

Acetic acid, mL

\begin{tabular}{lll}
\hline 1.6 & 4.5 & 9 \\
\hline
\end{tabular}




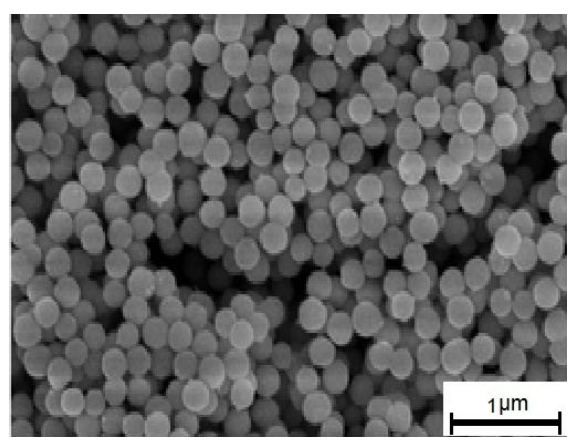

Figure 1. SEM scans from particles synthesized with generic sol-gel method.

\section{Changing the reaction conditions}

\section{Type of acid}

Three parallel reactions was performed according sol-gel method and adding, hydrochloric acid, nitric acid and Glacial acetic acid respectively to the reaction (Table 2). Formed products were excluded from the reaction environment in accordance with methods stated above and their particle size and morphology were examined by SEM. Figure 2 indicates the SEM scans of obtained products from this reactions.

Table 2. Using of Various Acids in sol-gel reaction.

\begin{tabular}{cccc}
\hline Acid type & Acid, $\mathrm{mL}$ & TEOS, $\mathrm{mL}$ & Deionized water, $\mathrm{mL}$ \\
\hline Nitric acid & 9 & 4.5 & 1.6 \\
Hydrochloric acid & 9 & 4.5 & 1.6 \\
Acetic acid & 9 & 4.5 & 1.6 \\
\hline
\end{tabular}
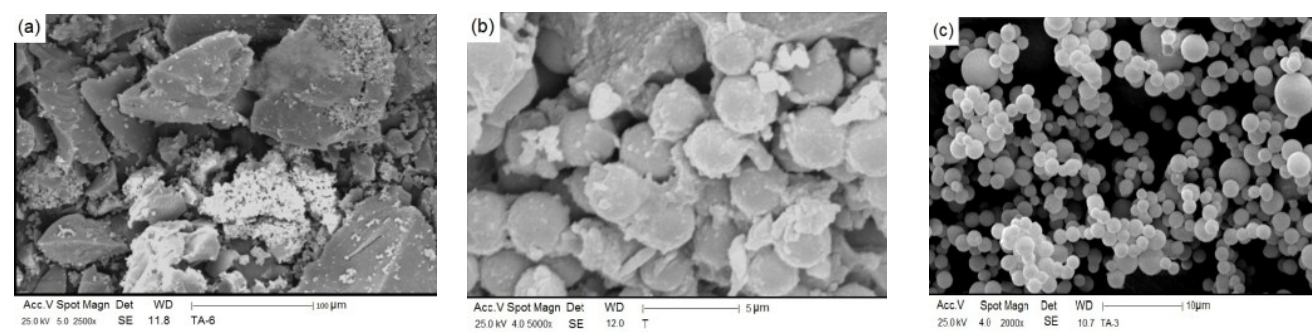

Figure 2. Synthesized particles in presence of various Acids: a) Nitric, b) Hydrochloric, c) Glacial Acetic.

\section{Change the ratio of acid}

To study the effect of changes in acid ratio on the size and morphology of particles, in eight parallel reactions $2,4,8,10,12,15,17.5$ and 20 ratios of the acetic acid in comparison with TEOS that was $2.4,4.7,9.37,11.72,14.1,17.6,20.5$ and $23.4 \mathrm{ml}$ of acid respectively were added to the reaction environment (Table 3). Formed products were removed from the environment and their particle size and morphology were examined by SEM. 
Table 3. Variation of acid volume in sol-gel reaction.

\begin{tabular}{|c|c|c|c|}
\hline Acid Ratio & Acid, $\mathrm{mL}$ & TEOS, $\mathrm{mL}$ & Deionized water, $\mathrm{mL}$ \\
\hline 2 & 2.4 & 4.5 & 1.6 \\
\hline 4 & 4.7 & 4.5 & 1.6 \\
\hline 8 & 9.37 & 4.5 & 1.6 \\
\hline 10 & 11.72 & 4.5 & 1.6 \\
\hline 12 & 14.1 & 4.5 & 1.6 \\
\hline 15 & 17.6 & 4.5 & 1.6 \\
\hline 17.5 & 20.5 & 4.5 & 1.6 \\
\hline 20 & 23.4 & 4.5 & 1.6 \\
\hline
\end{tabular}

\section{Change the ratio of TEOS}

To study the effect of acid ratio changing on particles properties, in five parallel reactions, the water and acetic acid ratio are considered and TEOS with ratio $1,0.9,0.8,0.6,0.4$ that were equal to value $4.5,4,3.6,2.7,1.8 \mathrm{ml}$ of TEOS, was added to reaction environment (Table 4 ). The obtained products were separated and their particle size and morphology were examined by SEM.

Table 4. Various TEOS molar ratios in sol-gel reaction.

\begin{tabular}{cccc}
\hline TEOS ratio & TEOS, $\mathrm{mL}$ & Deionized water, $\mathrm{mL}$ & Acid, $\mathrm{mL}$ \\
\hline 0.4 & 1.8 & 1.6 & 9 \\
0.6 & 2.7 & 1.6 & 9 \\
0.8 & 3.6 & 1.6 & 9 \\
0.9 & 4 & 1.6 & 9 \\
1 & 4.5 & 1.6 & 9 \\
\hline
\end{tabular}

\section{To change the reaction period}

Five parallel reactions were achieved with retention time 30 minutes, 60 minutes, 6 hours, 24 hours and 1 weekend and after considered time, the products were separated from reaction environment and were studied (Table 5).

Table 5. Change the retention time of sol-gel reaction.

\begin{tabular}{cccc}
\hline TEOS, $\mathrm{mL}$ & Acid, $\mathrm{mL}$ & Deionized water, $\mathrm{mL}$ & Retention time, $\mathrm{h}$ \\
\hline 4.5 & 9 & 1.6 & 0.5 \\
4.5 & 9 & 1.6 & 1 \\
4.5 & 9 & 1.6 & 6 \\
4.5 & 9 & 1.6 & 24 \\
4.5 & 9 & 1.6 & 168 \\
\hline
\end{tabular}

\section{Results and Discussion}

The examination of the change the type of acid effect on the particle size and morphology

In synthesized samples with nitric acid (Figure 2-a) particles were formed in a regular and irregular structure. It is due to the high concentration of ions in the environment that increases the ionic strength of solution. In this condition the primary cores are rapidly 
formed and then the core diameter increases very quickly due to high concentrations of ions in the environment in a way that the particles stick together and outer layers sink together and make some big hunk that a single particles cannot be seen and separated in any way. Particles performed from reactions in the presence of Hydrochloric acid, are observed in Figure 2-b. In this way, particles are detected separately, but the outer layers of composed particles sink together again due to the high concentration of ions in the environment. Hydrochloric acid has less ionic strength compared with nitric acid and therefore concentration of ions in the presence of this acid compared to nitric acid is less. This reduces the speed and range of nuclei growth process which is leading to sinking of particles lower together. But in the presence of acetic acid which has less acidic constant in comparison with the two previous acids, less concentration of ions is produced. In consequences, the products obtained are seen as spherical and separated grains in the presence of acid (Figure 3 ). These particles have the same dimensions. But this problem is controllable and removable with controlling the other variables.

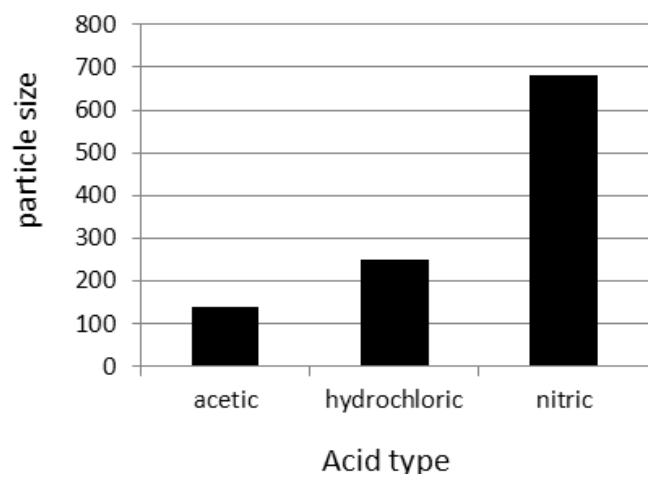

Figure 3. Change in particle size in presence of different acids.

\section{Change the acid ratio}

Because changing the ratio of acid, the ionic strength in reaction environment and consequently hydrolysis rate is changed, the size and shape of particles will also change. At lower ionic strength (the acid lower rate) condensation stage, is less complicated and therefore ionic layer formed around the particle has less diameter at this stage and therefore particle diameter will be less. As seen in Figure 4 ( $a, b$ and $c$ ) formed by the ratio of acetic acid, with increasing ratio of acid, the reaction rate of both hydrolysis and condensation increases. Consequently, at the beginning of the reaction, the core high-speed creation begins to advance that causes very high creation of the initial cores. Shortly after the start of the hydrolysis reactions, condensation reactions as well as competition started to do that the result of particle growth and formation of primary and secondary ion layers around them. In high ionic strength condensation cannot operate fully, resulting in the formation to complete particles with different diameters (Figure 5).

In these conditions, the adhesion of particles is also seen frequently and usually small cores, stick to larger particles in the condensation step. So with increasing the acid ratio, the 
percentage of clog up of the formed particles and forming two and multi nuclear particles increases (Figure 6).

But in low ratios of acid (i.e. 2, 4 and 8) grains are spherical and nearly the same size. Also reaction period by increasing of reaction period decreases frequently (Figure 7).
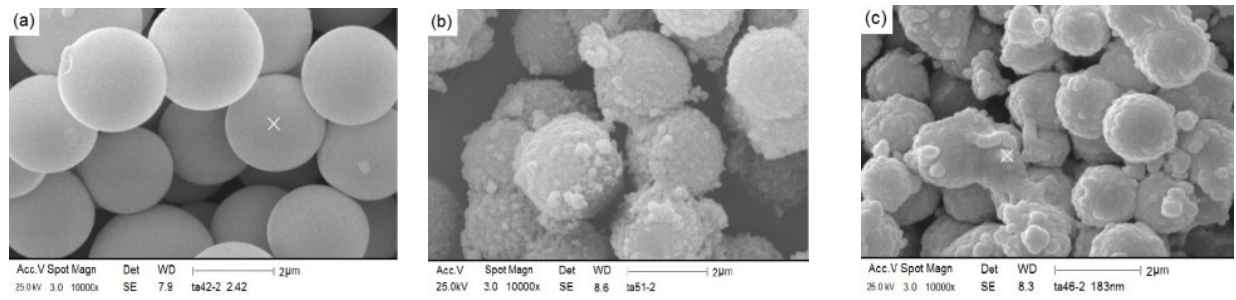

Figure 4. Particles synthesized in presence of different acid ratios: a) ratio $=4, b$ ) ratio $=10$, c) ratio $=20$.

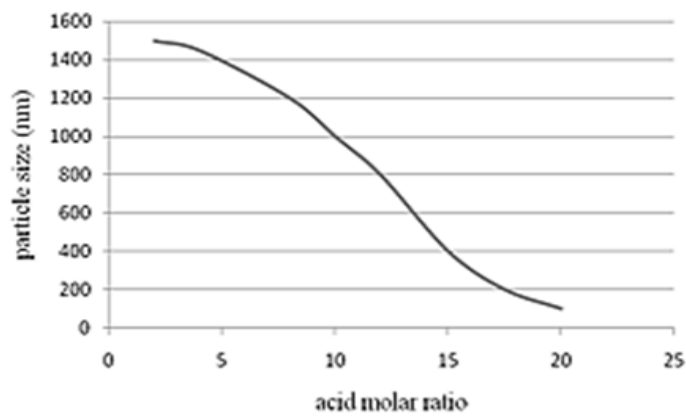

Figure 5. Particle size variations in different molar ratios of acetic acid.

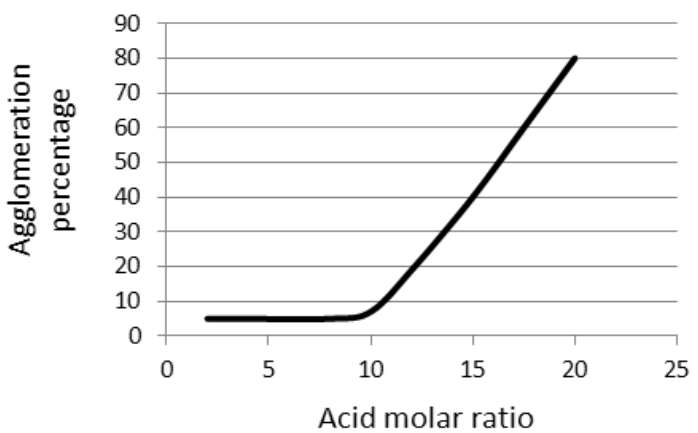

Figure 6. Relation between acid molar ratio and agglomeration of particles. 


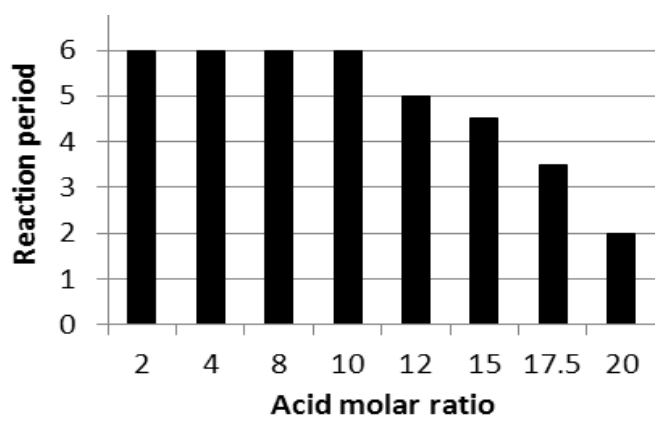

Figure 7. Relation between acetic acid molar ratio and reaction period Examining of adding small amounts of Lanthanum nitrate as structural modifier.

\section{To change the Tetraethylorthosilicate}

In the reaction achieved in TEOS with the ratios lower than 0.8 , the product formed was very rare. Figure 8 indicates the TEM pictures that their approximated size is 50 Nanometers. These pictures approve particle size. This is because of reducing the primary cores. This reduction has direct relation with TEOS to acetic acid ratio. The particles were separable and single particles were made and had equal size. This is same in the ratio 0.9 and 1 and separable particles with equal size that are spherical are made as shown in Figure 9. The particle sizes of products made in the reaction from every three stages are equal. As shown in Figure 10, particle size increased while TEOS molar ratio increases.

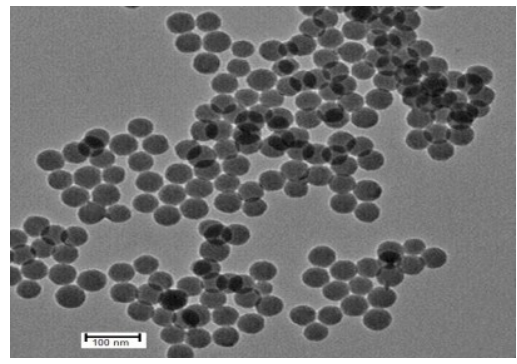

Figure 8. TEM scans from particles synthesized with 0.8 TEOS molar ratio.
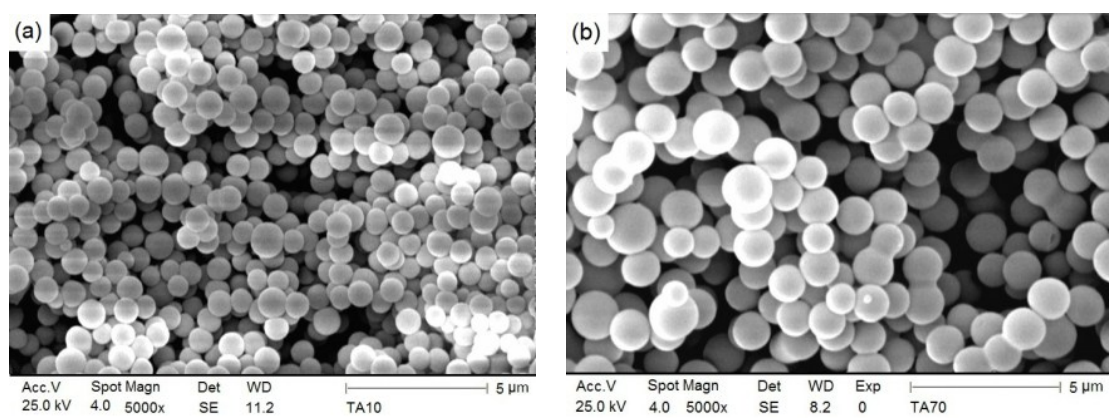

Figure 9. Synthesized particles in presence of different TEOS molar ratios: a) 0.9, b) 1 . 


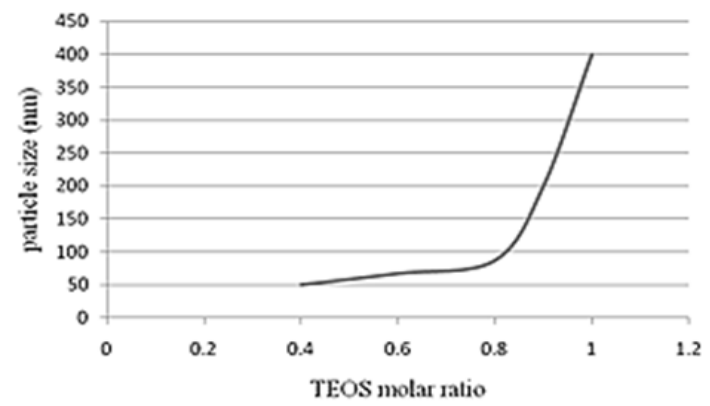

Figure 10. Particle size variation in presence of different TEOS molar ratios.

\section{Varying the time of the reaction}

In the beginning of reaction, the particles had smaller size and their morphology was not interesting because core creation was not completed and was continuing. Also the particle stability in the smaller particle is less and after spending a few hour of reaction beginning, core creation was completed and the layers around the particles were created and were continuing.
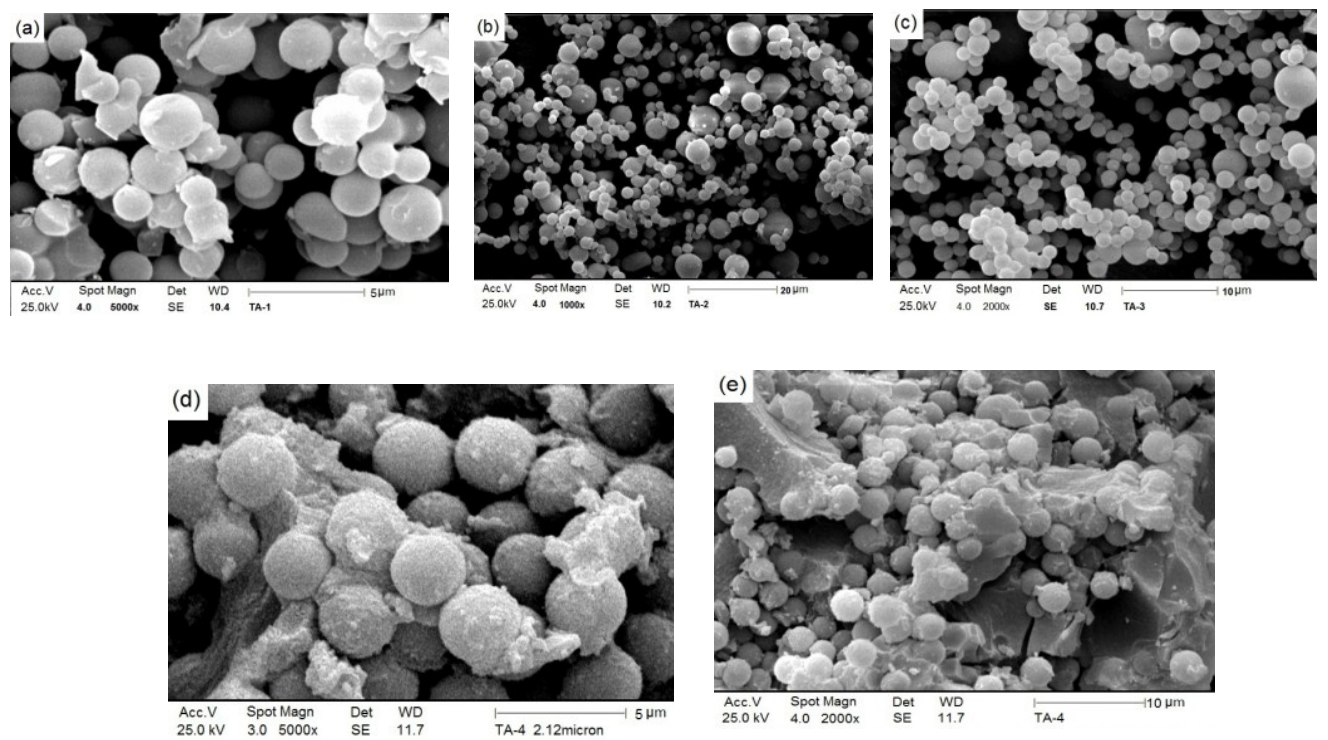

Figure 11. Synthesized particles produced in different reaction periods: a) 30 min, b) $60 \mathrm{~min}$, c) $6 \mathrm{~h}$, d) $24 \mathrm{~h}$, e) 1 weak.

Therefore, the particle had bigger size and very proper morphology, therefore, depending on size and that if the morphology in the product is important, the time of the reaction can be determined. As indicated in Figure 11.a, (after 30 minutes from beginning of the reaction), it 
was achieved primary core creation after spending this time and the growth was continuing. The particles had very different sizes and very various shapes .In the Figure 11-b in which the particles were assessed after 1 hour, the particles was spherical, the sizes were very various. In this stage, the speed of core creation speed was become lesser and the prevalent process in this stage was particle growth. Therefore, the secondary layers around the particles was made and some particles were grown and were became bigger because the condensation process was not completed and primary kernels were made and grown that causes to eliminate steady particles. As indicated in Figure 11-c the particle had same shape and were spherical and the size of the particles were same after 6 hours. As indicated in Figure 11-d, the two or three core particles were made after 24 hours and 1 weeks because the ionic concentration in the reaction was high and there was not any modifier to modify ionic concentration. Along condensation process, the particles were adhered and made. After this optimum time, the doesn't reacted reagents present in the reaction environment act as cement bed and caused to limit the particles in this bed.

\section{Conclusion}

In synthesized samples with nitric acid, particles are completely irregular and with no form structures. That is due to the high concentration of ions in the environment. Products obtained in this reaction cannot be used as a drug delivery system. Using the appropriate structural modifier adjusts ionic strength of environment and better control over size and morphology of the products can be applied. Products obtained in reaction with hydrochloric acid contain separable particles, but with poor morphology and in cases, including two and multi-core particles. This reaction can also use different concentrations of structural modifiers to control of size and morphology of particles produced. But in the presence of acetic acid that in comparison with the two previous acids have less acidic power, less concentration of ions is produced. Consequently, the products obtained in presence of this acid are seen as separated spherical grains. These particles have not the same dimensions. But the problem is controllable and removable with the control of other variables. In reactions that the acetic acid ratio is varied from 10 to 20 (compared to water) the reaction rate of both hydrolysis and condensation reactions increases due to high ionic strength in environment, with increasing ratio of acid. Consequently, at the beginning of the reaction, the high-speed core creation begins to complete that result to creation of huge number of initial cores. Shortly after the start of the hydrolysis reactions, condensation reactions started as a competition reaction that the particle growth and formation of primary and secondary ion layers are resulted around them. In high ionic strength condensation cannot operate completely, results in the formation of particles with different diameters. In these conditions, the adhesions of particles also are seen frequently and usually small cores stick to larger particles, in the condensation stage. Therefore, increasing the acid ratio, the percentage of clog up of formed particles and two and multi nuclear particles increase. In large acid proportions, it is suggested to use appropriate structural modifiers to control morphology and particle size. But in low ratios of acid, particles have the same size and nearly spherical. Particle size obtained in this reaction is between 1 and 2 microns. In the reactions achieved with TEOS with ratios lower than 0.8 compared water, the products are very rare because of reducing primary cores. This reduction is directly related with reducing TEOS to acetic acid ratio. As indicated in Figure 8, the particles obtained in ratio 0.8 of TEOS are disperse and separable and have same sizes. In this scan the size of obtain particles between 50 and 80 $\mathrm{nm}$. This is same in ratios $0.9,1$. The sizes of the particle in the products obtained of from three ratios are same and its average is 0.4 micron. Therefore, by using of ratio 0.8 for TEOS the industrial synthesis can be caused to reduce the final price. In the reaction in which the 
retention time is variable, the products have very different sizes after 30 minutes. These particles in drug delivery systems cause various problems that include non-proper size, twocore particles and different shapes. In particles obtained of $1 \mathrm{~h}$ retention time, the sizes are same and are among 0.5 and 1 micron. The particles are spherical this particles can be used in catalysis reactions. After 6 hours, the particles have same shape and are spherical and their sizes are same. Their sizes are among 0.5 and 1 micron and are dispersed. The sizes of the particles can be among 30 and $50 \mathrm{~nm}$ using of structural modifiers and copolymers. The products obtained in this reaction can be used to load radiotherapy drugs like lanthanides and used to therapy the liver cancer. Also, can control them with operating and doping the drug to them to emit the drug in the body.

\section{Acknowledgements}

This project was financial support by the Islamic Azad University, North Tehran Branch, of Iran.

\section{References}

1. Edimar D, Neri R.C, Osvaldo A.S, and Alexandreprado G.S, Chem Mater., 2007, 19(22), 5437.

2. Weber S.G, and Marsmann H, Nanoscale Science and Technology Series, Kluwer Academic Plenum Publisher, New York, Wehrspohn Ed R, 2005, 350.

3. Yingxu C, Tang R.L, Xinhua Y and Zhang P.X, Chinese $J$ of Chem Phys., 2008, 21(6), 596.

4. Samed A.J, Takayuki T, Keita I and Masato M, Topics in Catalysis., 53(7-10), 591.

5. Yan Q, Huanfa C, Yiyang L, Zhiyi Y, Xinhao C and Jianbin H, J Phys. Chem., 2011, 115 (15), 7323.

6. Pénard A, Gacoin and Boilot J, Acc. Chem. Res., 2007, 40(9), 895.

7. Casanova D, Giaume D, Boilot J.P and Alexandrou A, Biophys. Journal., 2007, 654.

8. Yu L, Alet P.J, Picardi G, Maurin I, Roca P and Cabarrocas I, Nanotechnology., 2008, 19, 485605.

9. Meijerink A, Wegh R, Vergeer P and Vlugt T, Optical Materials., 2006, 28(6-7), 575.

10. Pautrot D'alencon L, Barboux P and Boilot J.P, J of Sol-Gel Science and Technology., 2006, 39, 261.

11. Carriere D, Moreau M, Barboux P, Boilot J.P and Spalla O, Langmuir., 2004, 20, 3449.

12. Mritunjai S, Shinjini S, Prasada S and Gambhir I.S, Digest $J$ of Nanomaterials and Biostructures., 2008, 3(3), 115.

13. Moran C.E, Hale G.D and Halas N.J, with Rare Earth Dopants Langmuir., 2001, 17, 8376.

14. Dalili Mansour N, Zare K and Elsagh A, J of Phys and Theoretical Chem., 2010, 7(1), 15. 


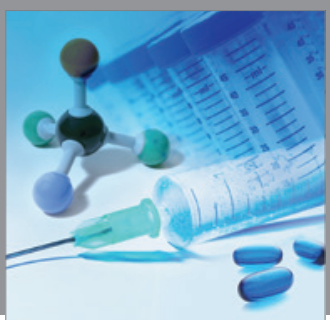

International Journal of

Medicinal Chemistry

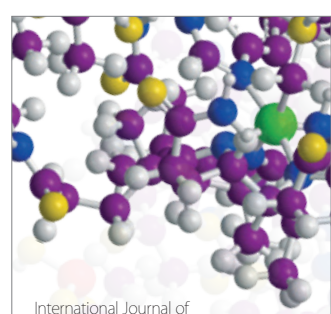

Carbohydrate Chemistry

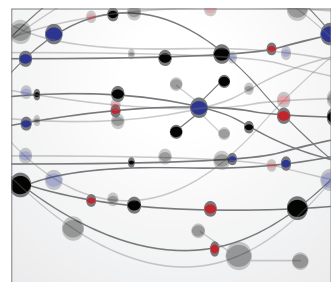

The Scientific World Journal
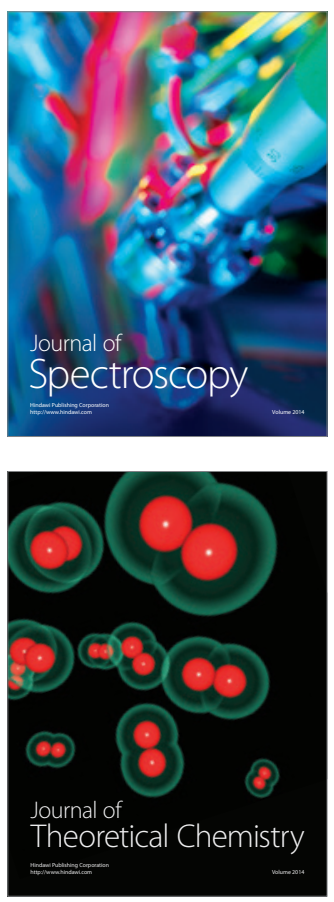
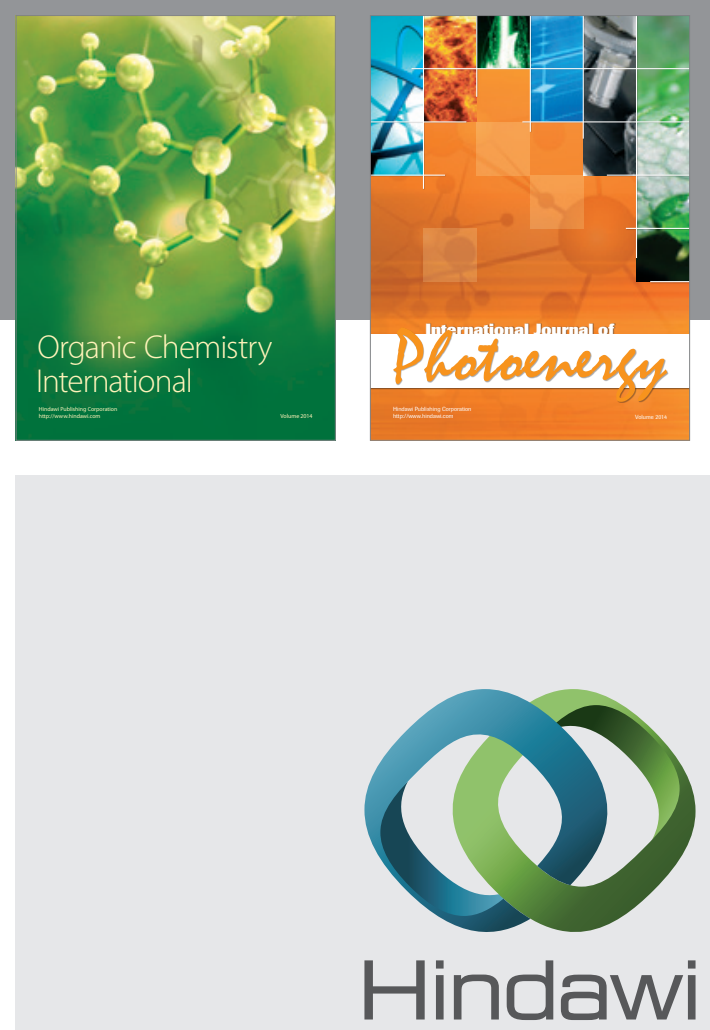

Submit your manuscripts at

http://www.hindawi.com
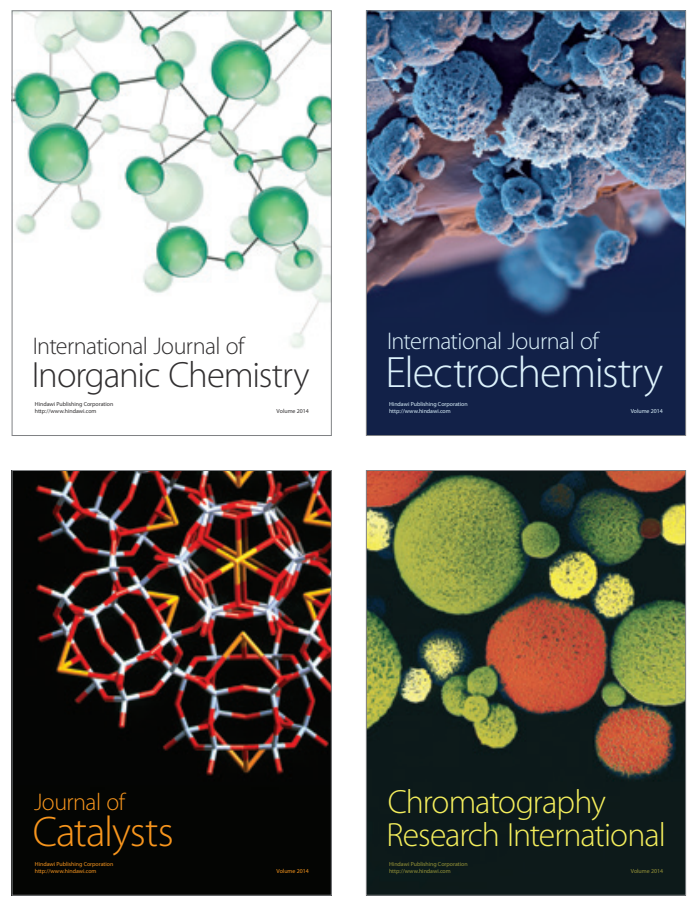
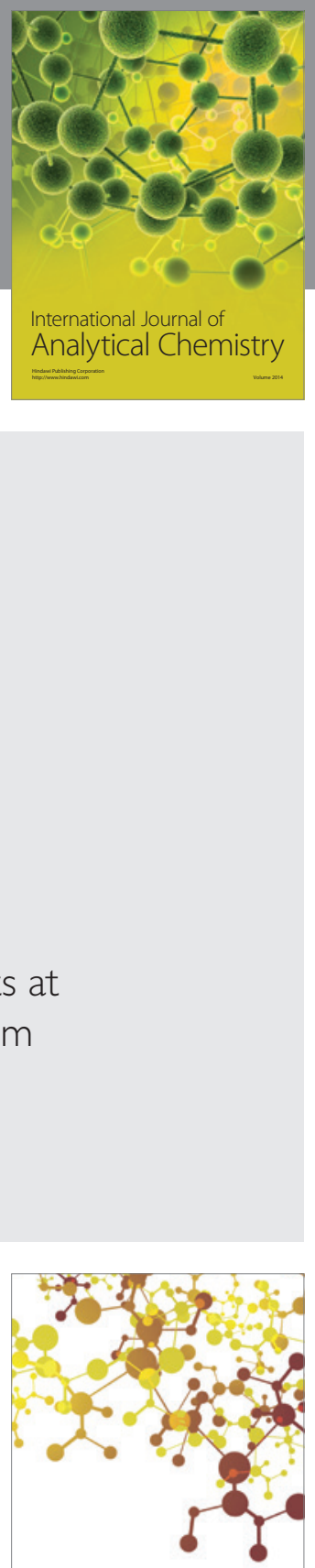

Journal of

Applied Chemistry
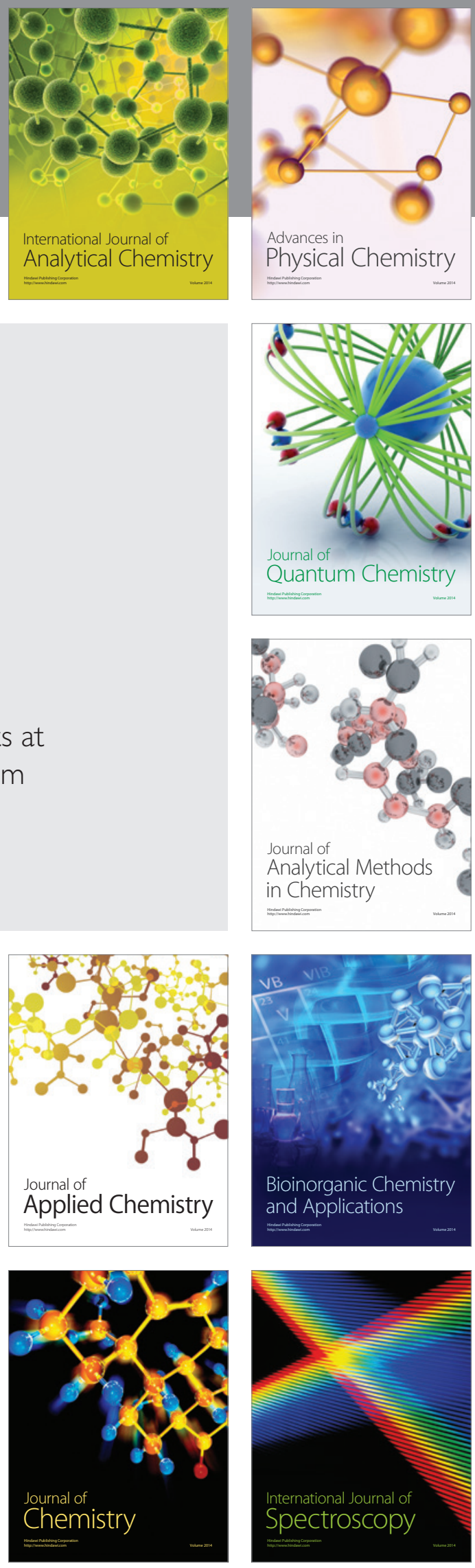